\title{
Sensor network integration for a medical device using CDG
}

\author{
Vasco Delgado-Gomes \\ DEE, FCT-NOVA \\ CTS-UNINOVA \\ Caparica, Portugal \\ vmdg@uninova.pt
}

\author{
Fábio Januário \\ DEE, FCT-NOVA \\ CTS-UNINOVA \\ Caparica, Portugal \\ faj@uninova.pt
}

\author{
Nuno Vilhena \\ DEE, FCT-NOVA \\ CTS-UNINOVA \\ Caparica, Portugal \\ nuv@uninova.pt
}

\author{
Ricardo Jardim-Gonçalves \\ DEE, FCT-NOVA \\ CTS-UNINOVA \\ Caparica, Portugal \\ rg@uninova.pt
}

\begin{abstract}
The weakness of the deep layer of lumbar or cervical spine muscles (so-called multifidus muscles) is among the most common causes for backpain. Frequent testing and measurements over a 20 years period of time showed a common weakness of this particular muscle group in more than $70 \%$ of investigated persons. Following a routine training has demonstrated an effective improvement in low back health. The Smart4Health project aims at empowering EU Citizens with an interoperable and exchangeable European Electronic Health Record (EHR) that will allow EU citizens to be active participants in managing their own health. As part of project activities, Smart4Health will upgrade existing physiotherapy devices with real-time measurements and connectivity capabilities, meeting new medical device regulations. Force and angle sensors measurement technology will be connected and integrated in the Smart4Health framework, enabling the patient with a real-time performance visualization and the treatment data upload for a secure cloud. The clinicians can then keep track of the patient treatment and made the necessary adjustments (if needed).The physiotherapy device and its sensors are connected over a secure network, enabling the data collection of health-related data. Moreover, an intelligent sensors network collecting citizen health and wellbeing data, adds information to citizen's EHR.
\end{abstract}

Keywords-Smart4Health, Continua Design Guidelines (CDG), Electronic Health Record (EHR), medical information systems, sensor network

\section{INTRODUCTION}

Smart4Health is a Horizon 2020 European Project aiming at empowering EU Citizens with an interoperable and exchangeable European Electronic Health Record (EHR) that will allow EU citizens to be active participants in managing their own health [1]. The key objective of Smart4Health is to place the Citizen in the centre of the decision of his / her Health data (Fig. 1).

The Smart4Health prototype will comprise the 4HealthPlatform (4HP) data layer connecting with the 4HealthNavigator $(4 \mathrm{HN})$ portal for services and applications to provide advanced personalized health services accessible whenever and wherever.

The research work leading to these results has received funding from the European Union's Horizon 2020 research and innovation programme under grant agreement No 826117- Smart4Health project. The sole responsibility of this publication lies with the author(s). The European Union and is not responsible for any use that may be made of the information contained therein.

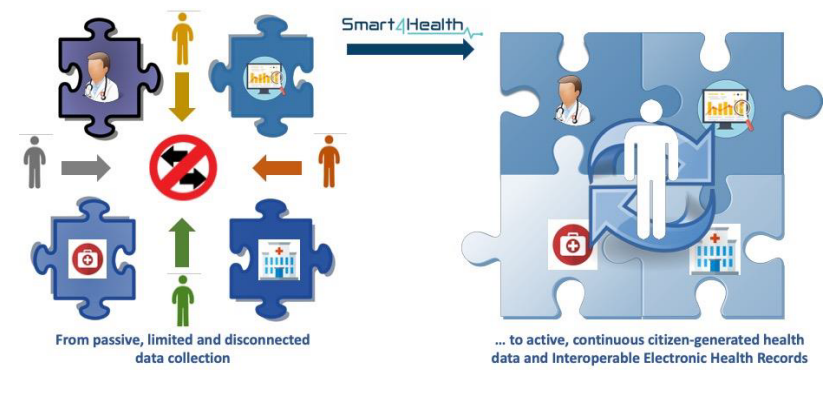

Fig. 1. The Smart4Health project goal: Empowering the citizen to manage own health data.

The 4HP, will empower citizens with electronic health(care) record exchange, personal connected health services, and the ability of data donorship to the scientific community. The 4HP will enable citizens to manage, collect, store, access and share own health and healthcare data with different clinicians and medical centres, with local and international societal and research activities and to cooperate directly with healthcare providers, through an easy-to-use, secure, constantly accessible and portable health data and services prototype within the EU and beyond.

To reach these objectives at European scale, the implementation strategy behind Smart4Health is based on a truly multidisciplinary approach. The project team is constituted by eighteen partners from seven different EU member states and the US, including: Technology and infrastructure Developers, Hospitals, Social Sciences Researchers, Physiotherapists, Nurses, informal caregivers, Regional Government, Research Centres, Universities and SMEs. Together with the technical development of the digital platform and tools, the project will actively involve the Citizens and User Communities that will be in the front line interacting with the technology.

The central role of the citizen is verified through the Fig. 1 and supported by 6 Use Design Cases (UDCs), namely:

- MyHealthView: Daily management, navigation, overview adapted for home, work, and when travelling.

- MyTrusted: Data sharing information with trusted ones - Patient Summary, Health status summary.

- Mob.E.Health: Sharing key information for quick under- 
standing of status, critical information, dynamic information that is relevant in unplanned situation and/or when needing medication.

- MyTime: Actual daily management - navigation, overview, like, interesting, informative, helpful.

- MyWork: Occupational Health, Safety and prevention at work activities and collection of citizens generated health data.

- MyScience: Donation for science and research.

In the UCDs the values, concerns and visions of users should be integrated along the co-creation principles. The possibility of data exchange, e.g. between citizens, different health professionals, research, will be considered and addressed for each UDC.

Citizens will be able to interact and test the different steps of Health Data Management, including Health Data collection, storage and usage during their daily life (at home, at work, while traveling, or during leisure and while practicing sport activities).

This paper describes the work under development for health data collection while using a physiotherapy device that will be equipped with smart sensors. The work is being developed following the Continua Design Guidelines principles. The data collected is sent over a network for the 4HP in order to be available anytime and anywhere both for the citizen and for the clinicians.

The physiotherapy device and its sensors will collect local data and forward it over the network to the 4HP using the CDG. This data composes the person's EHR and can be completed with other health-related data, such as data from fitness sensors and activity trackers.

In this context, a network composed of a diversity of intelligent sensors that track the citizen health and wellbeing is achieved in Smart4Health. Moreover, the citizen is enabled to manage and bridge their own data throughout the EU and beyond.

\section{Continua Design Guidelines}

The Personal Connected Health Alliance (PCHAlliance) Continua Design Guidelines (CDG) define an open and flexible framework of standards and criteria required to ensure the components interoperability used for applications monitoring personal health and wellness. They also improve interoperability by clarifying the underlying specifications and standards, reducing options or adding missing features [2]. For this purpose, CDG are aligned with other healthcare standard organizations, such as, Integrating the Healthcare Enterprise (IHE), Health Level 7 (HL7), ISO/IEEE and Healthcare Information and Management System Society (HIMSS) [3]. The CDG are recognized by International Telecommunication Union (ITU) as a formal international standard for personal health systems and currently support the integration of personal health data from 26 vital signs sensors and 40 health, medical, and fitness devices [4].

Fig. 2 shows the CDG high-level architecture enabling plugand-play connectivity of devices and services for personal health management and healthcare delivery. With these capabilities, CDG allow an easy and transparent connection of medical devices with gateways and end-service platforms. This architecture focuses on the interfaces described in the following subsections, namely: Personal Health Devices (PHDs) interface, Services interface, and Healthcare Information System (HIS) interface [5].

\section{A. Personal Health Devices Interface}

The PHD interface is the interface between a PHD and a Personal Health Gateway (PHG) that is standardized around the ISO/IEEE 11073 PHD family of standards for health data representation and exchange [7]. With this approach, the citizen knows what data was measured, where and how and that this critical information is not lost. Furthermore, the ISO/IEEE 11073 standards in CDG runs on top of Bluetooth, USB, NFC and ZigBee transport protocols [8]. Fig. 3 shows the ISO/IEEE 11073 family of standards with the supported devices specializations [9]. In addition, CDG also works with Bluetooth to ensure that Bluetooth low energy (LE) technology healthcare profiles are compliant with the ISO/IEEE 11073 data format [10].

\section{B. Services Interface}

The Services interface is the interface between a PHG and Health \& Fitness Service (HFS) that implements an open standard solution for uploading device observations, exchange of questionnaires and responses, consent management, capabilities exchange, and authenticated persistent sessions [6].

Device observations are point-to-point, one-way transmission of a measurement between a PHG and an HFS using HL7 V2.6 Observations payloads. This transmission can be implemented by an PCD-01 message packaged (IHE Device Enterprise Communication profile) in SOAP and authenticated using SAML, where the CDG allow the mapping of ISO/IEEE 11073-20601 attributes to a PCD-01 message and preserves the ISO/IEEE 11073 nomenclature [12]. HL7 V2.6 Observation payloads can also be uploaded using the HL7 FHIR Resources via REST and OAuth, where CDG allow the mapping of ISO/IEEE 11073-20601 attributes received from a device into FHIR Resources [13].

Questionnaires are used to collect information from the citizen to contextualize the observations coming from remote devices. Questionnaires are presented according to the HL7 Implementation Guide for Questionnaire Form Definition document (HL7 CDA QFD). Responses to a questionnaire are then presented according to the HL7 Implementation Guide Questionnaire Response document (HL7 CDA QRD) [2].

Consent management enables citizen to choose what heath data they are willing to permit their healthcare providers to access. The consent documents are exchange using hData over HTTP protocol. Consent enforcement is enabled using the IHE Document Encryption (DEN) profile [13].

Capability exchange in Services interface allows the reduction of information that must be pre-configured on a device in order to get pug-and-play capability. CGD define the properties 


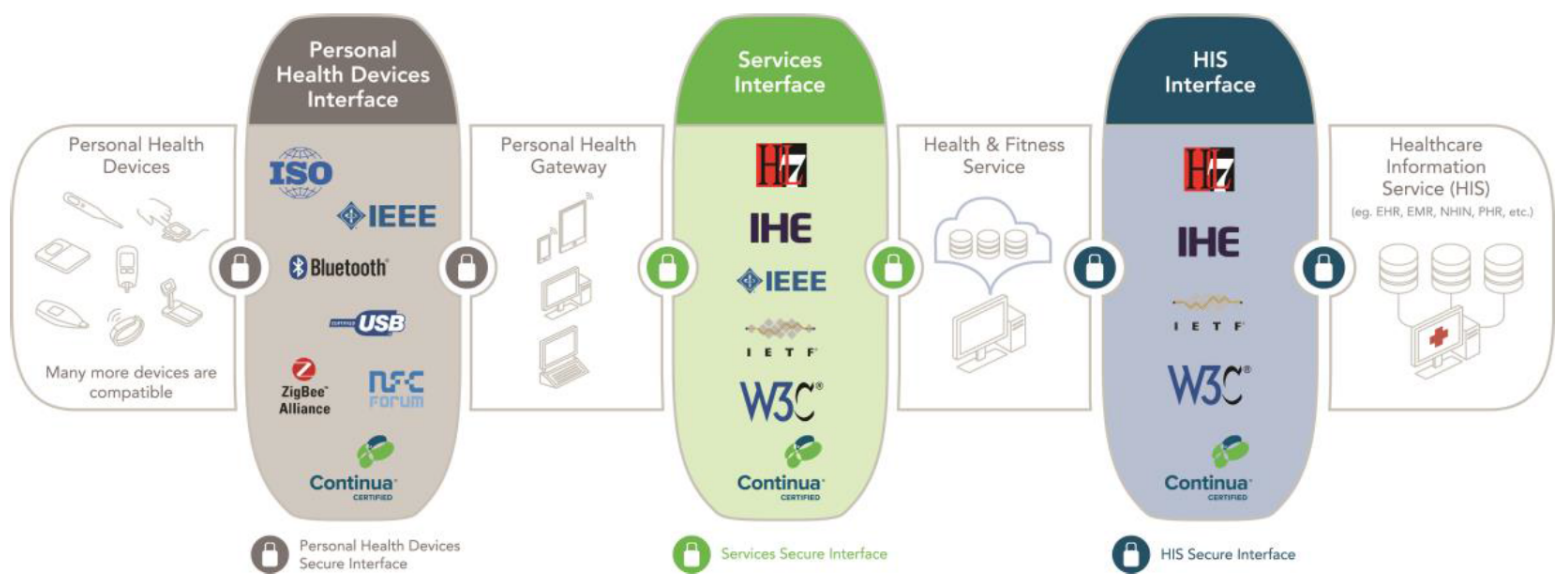

Fig. 2. Continua Design Guidelines architecture [6].

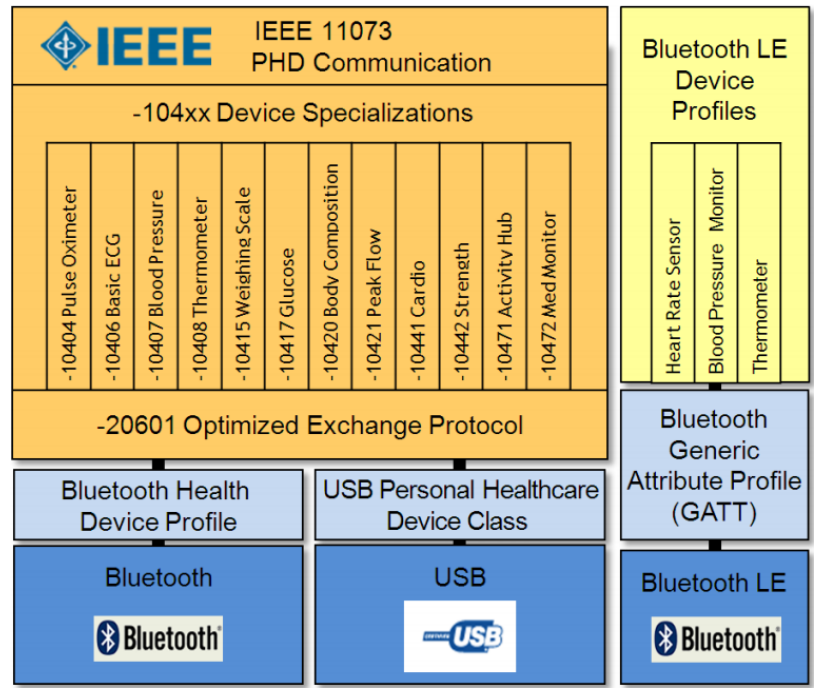

Fig. 3. PHD protocol stack [11].

of a device or service and how to start the exchange of this information. This information can be encoded in XML or JSON formats [6].

Authenticated Persistent Session (APS) enables a cloud service to have a persistent secure channel to a gateway in the cellular environment where bandwidth, power, and IP resources may be limited and/or intermittent. The APS uses RESTful exchanges to establish the communications channel and MQTT, a lightweight publish-subscribe based protocol standard, to exchange messages [13].

\section{Healthcare Information System Interface}

The HIS interface is the interface between an HFS and an HIS providing the electronic exchange of health records employing an HL7-based PHMR [14]. The PHMR is defined by HL7 to carry personal healthcare monitoring information to electronic medical record systems and includes representation of measurements captured by personal health devices.
These reports can be exchanged using IHE Cross-Enterprise Document Sharing (XDS) and IHE Patient Identifier CrossReference (PIX) profiles for cross-referencing patient identifiers and cross-enterprise document sharing. Moreover, ONC DIRECT can also be used providing a simple and secure standard-based method for sending health information to the known and trusted participants via email over the Internet [15].

\section{Physiotherapy Device}

Focusing on the problem of the weakness of lumbar or cervical spine muscles, which is the most common cause of backpain, a physiotherapy device can be used to train their deeper muscles, reinforcing them and helping to decrease the patient backpain. The training and the treatment consist on the active strengthening and stabilization of the entire spinal musculature through targeted, isolated exercises on medical devices, which will be upgrade with a force and angle sensor, providing isolated device measurements. The strengthening therapy of the back muscles targets the specific muscles, but neither the joints nor the discs loaded.

The physiotherapy device used in this project is a MedX device, by MedAix and it is shown in Fig. 4 [16]. Fig. 5 shows the conceptual diagram of the device which should highlight the following characteristics:

- Fixation for the legs and pelvis

- Isolation of the lumbar extensors

- Balance of upper body weight in gravity with the help of a counterweight

- Computer-assisted diagnostics and therapy

- Force and angle sensors

Fig. 5 also shows the patient position inside the physiotherapy device and the training movement that the patient should execute. The maximum extended position is $0^{\circ}$, the maximum flexed position is $72^{\circ}$ and the null position is $18^{\circ}$. This angle value is one of the two required measurements. The second measurement is the force executed by the patient when the training movement is executed. The duration of the training depends on the prescribed therapy. 


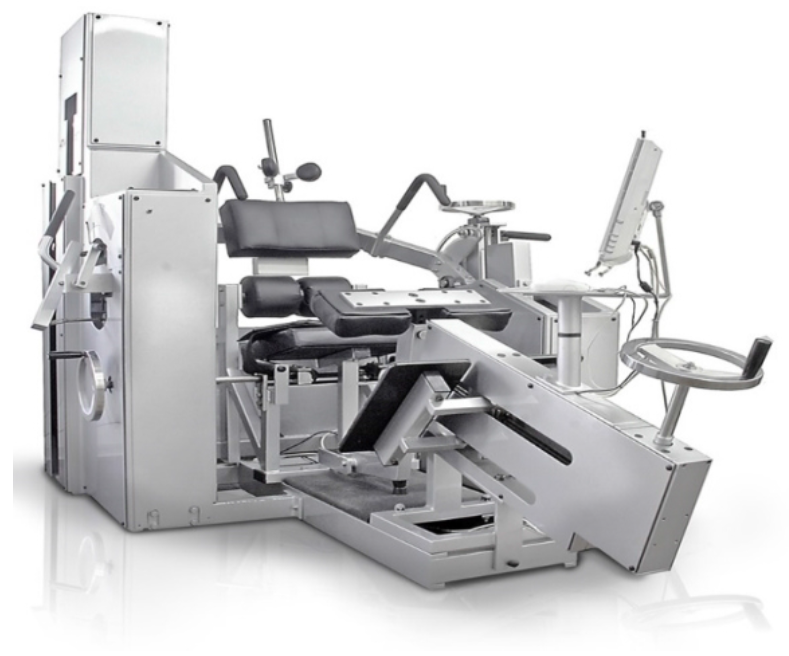

Fig. 4. Conceptual diagram of the sensors data acquisition.

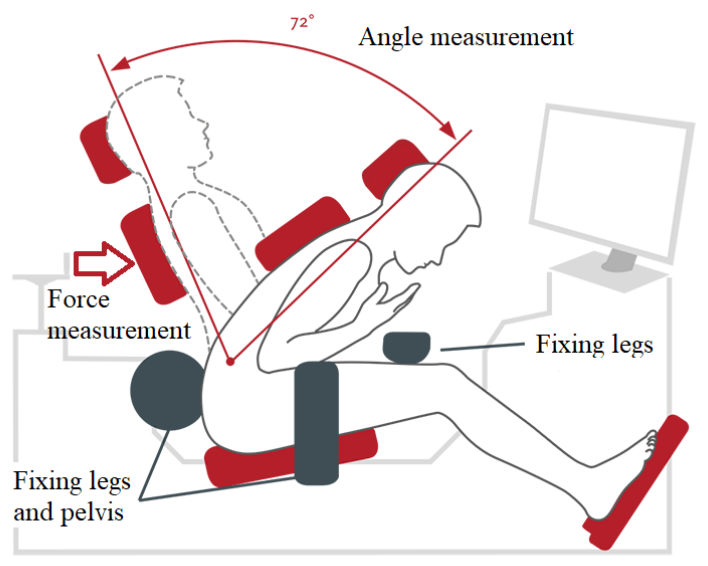

Fig. 5. Conceptual diagram of the physiotherapy equipment (adapted from [16].

The health data generated with this physiotherapy training device must be sent to the 4HP, therefore the equipment will be upgraded for connectivity, meeting the new medical device regulation, such as with individual Unique Device Identification (UDI) codifications.

\section{A. Device Measurements}

There are two main measurements in the physiotherapy machine:

- The angle of the movement executed by the patient while in training. The angle range is between $0^{\circ}$ and $72^{\circ}$.

- The force associated to the movement of the patient back. The force range is between 0 and $200 \mathrm{kgf}$.

\section{B. Sensors and Data Acquisition}

The sensor to measure the force executed by the patient with its back is a load cell, based on a strain gages bridge.
In order to acquire its signal, a signal conditioner amplifier is used, and measurement values are sent through the digital communication protocol $\mathrm{I}^{2} \mathrm{C}$ to the data acquisition board.

The sensor to measure the angle of the patient's back is a hall effect sensor. An Analog-to-digital converter (ADC) signal conditioner and the $\mathrm{I}^{2} \mathrm{C}$ protocol is also used.

The data from the sensors are acquired by the data acquisition board and are stored in a local buffer using the OAuth2 protocol, in order to be processed later (Fig. 6). Table I shows the specifications of the sensors installed in the physiotherapy device. These sensors data will be then forwarded to the 4HP.

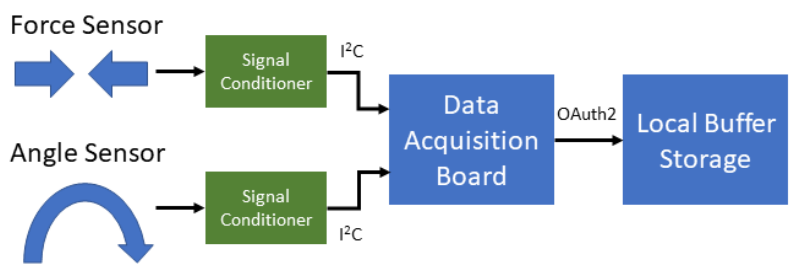

Fig. 6. Conceptual diagram of the sensors data acquisition.

TABLE I

Physiotherapy Device Sensors SPeCification

\begin{tabular}{|c|c|c|}
\hline Sensor & Description & Value \\
\hline & Model & Tedea-Huntleigh 615Steel \\
& Capacity & $200 \mathrm{kgf}$ \\
Load Cell & Excitation & $5 \mathrm{VDC}$ \\
& Resolution & $2 \mathrm{mV} / \mathrm{V}$ \\
& Output Impedance & $350 \pm 3 \Omega$ \\
& Type & Strain Gages Bridge \\
\hline \multirow{3}{*}{ Angle Sensor } & Model & TT Electronics 6127 Series \\
& Resolution & $180^{\circ}$ \\
& Type & Hall Effect \\
\hline
\end{tabular}

\section{SMART4HEALth INTEROPERABILITy AND INTEGRATION}

Smart4Health project aims to enable the integration of subsequent types of data such as quantified-self data or Omics data, with conventional healthcare data using the European EHR exchange format and standards. To assure interoperability and enable ingestion of citizen-generated/personal connected health data, Smart4Health adopts data exchange standards promoted by the PCHAlliance CDG. The integration of sensors in a physiotherapy device, that was described in section III, and the adoption of the exchange data architecture presented in Fig. 7 is one of these examples.

Fig. 7 shows the integration architecture of the physiotherapy device with the 4HP. In order to ensure the interoperability between the components, the standards suggested by CDG are used, namely a device interface and a service interface. In relation to the hardware, besides the physiotherapy device and underlying sensors already described, a laptop is used as a gateway (citizen integration HUB).

The physiotherapy device is connected to the citizen HUB via USB. The data transmission between the physiotherapy 

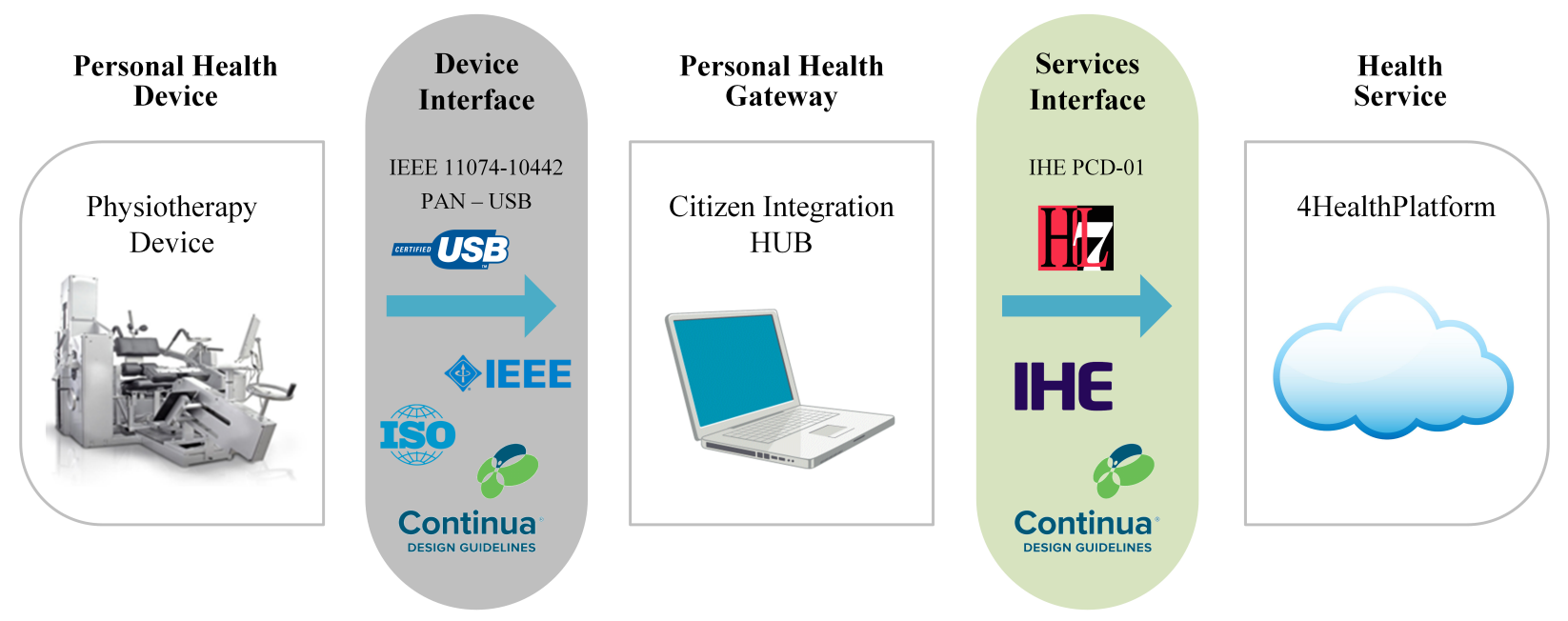

Fig. 7. Integration architecture of the physiotherapy device sensors with the 4HealthPlatform using the CDG.

device local buffer and the citizen HUB is done by the ISO/IEEE 11073-10442 (Strength fitness equipment) standard, since the exercises performed in the physiotherapy device and the data collected are compatible with strength equipment.

The citizen integration HUB allows the patient to locally see the exercises that are being performed, as well as important information and indications about them. This device also allows this data export to the 4HP, after the patient authorization.

The data transmission from the citizen HUB to the 4HP is done by a PCD-01 message as recommended by CDG. The harmonization and mapping of the existing nomenclature defined by the ISOO/IEEE 11073 standard to the PCD01 messages is done by the Rosetta Terminology Mapping (RTM). The PCD-01 messages, in addition to the sensors mapping data, also include fields for patient information.

Finally, the 4HP stores all citizen data, which can be visualized by 4HP. Future work includes the implementation of the HIS interface that will provide the electronic exchange of health records stored in the 4HealthPlatform.

The physiotherapy device generated data will be collected in the context of the MyHealthView UDC, which supports the collection of citizen-generated health data, covering: personal data and records, professional produced health records, medication, and other relevant information.

The collected data can then be shared with the patient permission to medical professionals (MyTrusted and Mob.E.Health UDCs), and scientific data donation (MyScience UDC).

\section{CONClusion}

One of most common causes of backpain is due to multifidus muscles weakness. One of the Smart4Health objectives is to decrease the sick leave days due to backpain, using a physiotherapy medical device to strengthen the back, both by training and prevention treatment sessions.

The physiotherapy device will be upgraded with angle and force sensors with the communication capability, to enable data exchange between the medical device and the clinician, through the 4HP. This physiotherapy device will be deployed in Germany, Luxembourg, and Portugal.

As future work, fitness health data will be also gathered and stored in the 4HP using the CDGs in a similar approach as used in the physiotherapy device information.

\section{REFERENCES}

[1] Smart4Health, "Citizen-centred EU-EHR exchange for personalized health," https://www.smart4health.eu/, January 2019, (Accessed 13March-2019).

[2] Continua Design Guidelines, "H.810 Introduction - Interoperability Design Guidelines for Personal Connected Health Systems," Personal Connected Health Alliance, Tech. Rep., 2017.

[3] M. Frohner, P. Urbauer, M. Forjan, B. Pohn, F. Gerbovics, S. Sauermann, and A. Mense, "Development of an Android App in Compliance with the Continua Health Alliance Design Guidelines for Medical Device Connectivity in mHealth," Biomedical Engineering / Biomedizinische Technik, vol. 57, no. SI-1 Track-N, jan 2012. [Online]. Available: https://www.degruyter.com/view/j/bmte.2012.57.issue-s1-N/ bmt-2012-4203/bmt-2012-4203.xml

[4] M. L. Braunstein, Health Informatics on FHIR: How HL7's New API is Transforming Healthcare. Springer, 2018.

[5] D. F. Santos, H. O. Almeida, and A. Perkusich, "A personal connected health system for the Internet of Things based on the Constrained Application Protocol," Computers \& Electrical Engineering, vol. 44, pp. 122-136, 2015.

[6] Continua Design Guidelines, "Fundamentals of Medical-Grade Data Exchange," Personal Connected Health Alliance, Tech. Rep., 2018.

[7] Continua Design Guidellines, "H.811 Personal Health Devices Interface design guidelines," Personal Connected Health Alliance, Tech. Rep., 2017.

[8] M. Benner and L. Schope, "Using continua health alliance standards - implementation and experiences of ieee 11073," in 2011 IEEE 12th International Conference on Mobile Data Management, vol. 2, June 2011, pp. 40-45.

[9] K. Han, M. Jung, and J. Cho, "Implementation of the personal healthcare services on automotive environments," Personal and Ubiquitous Computing, vol. 18, no. 3, pp. 523-533, Mar 2014.

[10] G. Lamprinakos, S. Asanin, T. Broden, A. Prestileo, J. Fursse, K. Papadopoulos, D. Kaklamani, and I. Venieris, "An integrated remote monitoring platform towards Telehealth and Telecare services interoperability," Information Sciences, vol. 308, pp. 23-37, 2015.

[11] D. Zhong, M. Kirwan, and X. Duan, "Developing interoperability standards for personal health devices," https://www.who.int/medical $\backslash$ _devices/global $\backslash$ forum/Sun $\backslash \_$pm $\backslash \_S A F \backslash 3 \backslash$ ZHONG.pdf, November 2013, (Accessed 13-March-2019). 
[12] P. Urbauer, S. Sauermann, M. Frohner, M. Forjan, B. Pohn, and A. Mense, "Applicability of IHE/Continua components for PHR systems: Learning from experiences," Computers in Biology and Medicine, vol. 59, pp. 186-193, 2015 .
[13] Continua Design Guidelines, "H.812 Services Interface Common Design Guidelines," Personal Connected Health Alliance, Tech. Rep., 2017.

[14] F. Wartena, J. Muskens, and L. Schmitt, "Continua: The impact of a personal telehealth ecosystem," in 2009 International Conference on eHealth, Telemedicine, and Social Medicine, Feb 2009, pp. 13-18.

[15] Continua Design Guidelines, "H.813 Healthcare Information System (HIS) Interface," Personal Connected Health Alliance, Tech. Rep., 2018.

[16] MedAix, "MedX-Therapie," https://www.medaix.de/physiotherapie/medxtherapie, June 2017, (Accessed 13-March-2019) 